Guegia-Hurtado, J.P. (2017). Al paso de las semillas de vida del pueblo nasa de Tierradentro y la construcción de las nuevas identidades. Revista de Antropología y Sociología: VIRAJES, 19 (2). 11-30. DOI: 10.17151/rasv.2017.19.2.2

\title{
AL PASO DE LAS SEMILLAS DE VIDA DEL PUEBLO NASA DE TIERRADENTRO Y LA CONSTRUCCIÓN DE LAS NUEVAS IDENTIDADES*
}

\author{
JINA PAOLA GUEGIA-HURTADO**
}

Recibido: 30 de abril 2017

Aprobado: 27 de julio de 2017

\author{
Artículo de Investigación
}

\footnotetext{
* Este artículo es una modificación de la ponencia presentada en la II Bienal de Infancias y Adolescencias desarrollada en Manizales en noviembre de 2016. Es un resultado parcial del proyecto de investigación titulado: "Prácticas de identidad en la diversidad en los procesos de socialización y subjetivación de las y los jóvenes del resguardo de Mosoco, Páez, Cauca".

** Magíster en Educación y Desarrollo Humano. Universidad de Manizales. Manizales, Colombia. E-mail: jinaguegia@gmail.com. (1) ORCID: 0000-0002-4434-8231. Google Scholar
} 


\title{
Resumen
}

Objetivo. Mostrar cómo niños y niñas del resguardo indígena de Mosoco construyen escenarios que promueven el buen vivir para sus familias y comunidad mediante la adquisición de aprendizajes colectivos, reflexiones y vivencias del contexto que habitan. Metodología. Se da cuenta de ello a través de la experiencia en campo durante la formulación de la política pública de infancia niñez y juventud del municipio de Páez, así como el reconocimiento de escenarios construidos por los participantes durante la propuesta educativa para el agenciamiento social (PEPAS). Resultados. En este sentido se da un acercamiento inicial a algunos momentos de los procesos de socialización y construcción del individuo a partir de apartados que resaltan la familia, la escuela, la comunidad y otros que hacen parte del proceso de construcción de identidades individuales y colectivas de los jóvenes. Con lo anterior se encuentran puntos de lectura de la compresión de las nuevas prácticas de identidad en los procesos de socialización y subjetivación de las nuevas generaciones; pero también de las dinámicas comunitarias que se tejen en torno a los procesos actuales a nivel político, económico y cultural. Conclusiones. Finalmente se hace necesario reflexionar sobre el papel de las comunidades indígenas, considerando la atención a las semillas de vida como agentes y actores sociales, buscando recuperar sus miradas en los entramados de relaciones culturales; ya que estos son la base de una buena educación y la generación de condiciones para la creatividad, la recreación y el relacionamiento con la madre naturaleza, el entorno social y un buen vivir para todos desde un contexto diverso.

Palabras clave: identidad, prácticas, diversidad, política, comunidad.

\section{WITH THE PASSAGE OF THE TIERRADENTRO NASA PEOPLE SEEDS OF LIFE AND THE CONSTRUCTION OF NEW IDENTITIES}

\begin{abstract}
Objective: The purpose of this article is to show how children from the Mosoco indigenous shelter construct scenarios that promote good living for their families and community through the acquisition of collective learning, reflections and experiences of the context they inhabit. Methodology: This is carried out through field experience during the formulation of the Public Policy on Childhood and Youth of the Municipality of Páez, as well as through the recognition of scenarios constructed by the participants during the educational proposal for social agency. (EPSA). Results: In this sense, there is an initial approach to some moments of the socialization and construction processes of the individual, starting with sections that highlight the family, the school, and the community among others that are part of the process of building individual and collective identities
\end{abstract}


and of young people. With the above, points of reading to understand the new practices of identity in the processes of socialization and subjectivation of the new generations are found, but also of the community dynamics that are woven around the current political, economic and cultural processes. Conclusions: Finally, it is necessary to reflect on the role of indigenous communities, considering the seeds of life as agents and social actors, seeking to recover their views in the maze of cultural relations, as they are the basis of a good education and the generation of conditions for creativity, recreation and relationship with mother nature, the social environment, and in general, a good living for all from a diverse context.

Key words: identity, practices, diversity, politics, community.

\section{Introducción}

1 resguardo indígena de Mosoco hace parte de la organización indígena regional del departamento del Cauca, por lo que a través de esta participación se interpretan los procesos de socialización que reciben los niños mediante un ejercicio social que fundamenta los intereses políticos y de resistencia cultural. Al ser este un interés fundamental para el fortalecimiento de la comunidad, se concibe como los actores interiorizan modelos y marcos colectivos percibidos por los niños y jóvenes a través de prácticas e imaginarios apuntados hacia el buen vivir (weçxweçxa) ${ }^{1}$.

Teniendo en cuenta lo anterior, la primera parte expone brevemente algunas conceptualizaciones sobre socialización dentro de un marco teórico $\mathrm{y}$ en escenarios en los que se desenvuelven las futuras generaciones. A partir de esta localización analítica son leídos los ciclos de vida nasa que enmarcan la comunidad, atravesada por unas líneas de acción, bajo un contexto nutrido por otros pueblos que también hacen parte de factores externos que inciden con gran influencia sobre los jóvenes.

Para ello, la segunda parte expresa la experiencia significativa a través de la construcción de la política pública de infancia niñez y juventud realizada en el municipio de Páez. Con base en esta herramienta de lectura se abordan las prácticas de identidad tenidas en cuenta por los niños, principales actores, quienes habitan en un territorio pluricultural que

\footnotetext{
${ }^{1}$ Buen vivir en armonía-equilibrio con la naturaleza, con los seres tangibles e intangibles.
} 
busca la convivencia armónica de sus habitantes a través del respeto por la diferencia.

Por otrolado, se da cuenta del proceso de diálogo en la implementación y discusión de la propuesta educativa para el agenciamiento social; allí, con la comunidad educativa de la institución educativa Juan Tama, se centra en las prácticas que generan tensión por las formas de ser y estar de los escolares dado que incorporan en la lectura de sus realidades distintos procesos de subjetivación. Finalmente se concluye, señalando la importancia de una educación pensada en las "semillas de vida" ${ }^{2}$ como principales actores y reproductores de prácticas propias y del fortalecimiento cultural; sin dejar de lado contextos diversos para tejer estrategias entre los ámbitos educativos, políticos, económicos, que se inscriban en los planes de vida de los resguardos.

\section{Algunos apuntes respecto a la socialización}

Cuando un individuo llega a este mundo, incluso antes de su nacimiento, inicia un proceso de aprendizaje y transición frente a lo que construye para sí mismo en relación con los demás; de esta manera los esquemas cognitivos, su comportamiento y modo de ser, son leídos a partir de los hábitats que recorre durante toda la vida hasta su muerte. Podríamos decir que es una secuencia temporal, en cuyo curso los habitantes que allí existen son inducidos a participar en la dialéctica de la sociedad, que implica el descubrimiento de la realidad cultural y —en relación dialéctica con ella- la construcción de la identidad personal.

Para el caso de las comunidades indígenas, por ejemplo, la tierra, la cosmovisión, el idioma, la espiritualidad o el apego a la naturaleza Giménez, (2002), como citó en Bello, (2004), se convierten en la base de entrada a la vida de todo ser nasa; elementos que giran en torno a los espacios de socialización que construyen los jóvenes hoy, sin quitarle la lectura del entorno diverso $u$ otros grupos humanos que acompañan esa transformación y el descubrimiento de una pluralidad de modos de vivir y entender la realidad. Por tal motivo la socialización puede ser vista como un proceso de construcción social de la realidad, dando cuenta de las diversas etapas de las que hablaremos a continuación.

\footnotetext{
2 "Semillas de Vida", son la traducción al español de la manera como en nasa se anuncia a los niños y niñas.
} 


\section{Socialización primaria}

Es la primera por la cual el individuo atraviesa en la niñez. Por medio de ella se convierte en miembro de la sociedad, semejante a la tulpa, entendida como un lugar inicial de encuentro donde se da fuerza al acompañamiento de los abuelos en relación a la trasmisión de costumbres, usos y prácticas que envuelven la tradición oral mediante cuentos, mitos y relatos acogidos por las nuevas generaciones; las cuales interiorizan, reproduciendo el orden social en el curso mismo de su acción, como se denota, poniendo a la socialización como una herramienta que 'formatea' a los individuos de modo que se conduzcan — sin que a menudo ellos mismos lo sepan- conforme a las necesidades del sistema (Lockwood, 1964).

Profundizando un poco más en el tema, podemos decir que el fogón es el centro de la casa entre los indígenas y los campesinos. En este se preparan los alimentos y brota el fuego para mantener el calor de la casa, de las personas y de los animales que se le acercan; por ello es el sitio de encuentro y de reunión de quienes la habitan, vienen de visita o llegan para encontrar hospitalidad. Alrededor del fogón se mezclan las risas de los niños con las angustias de los adultos y las memorias de los mayores. Congrega a los miembros de la familia y de la comunidad sentados en bancos de madera $o$ sobre la tierra entre penumbras, chispas del fuego y fogonazos de luz que se reflejan en los rostros de los presentes. Sus diálogos, críticas y chistes recrean diaria y permanentemente la vida del hogar, la comunidad y la organización (Espinosa Álzate, 2003).

Junto al fogón se retoman individualmente las decisiones de rechazo o de apoyo que son cruciales para cualquier acción de la comunidad o la organización. Aquí, se dice la palabra que no se ha dicho hasta el momento, se confiesa lo que no es público, se manifiestan sin temores las más íntimas apreciaciones, se interioriza y valora, se reafirman posiciones y convicciones, se amplía la consciencia porque se entiende lo que no había sido claro lo que no fue comprendido-y se complementa lo que se dijo o informó cuando no fue escuchado.

Con esta realidad entre manos una etapa posterior a la niñez comienza a interactuar más frecuentemente con entornos distintos a los de la familia, con personas distintas a sus padres o cuidadores habituales (Berger y Luckmann, 1995).

La vivencia con otros niños y con los maestros en el colegio da lugar a un nuevo modo de comunicación. Se empieza a aprender un amplio y diverso grupo de habilidades sociales y un conocimiento más detallado de los roles fuera de la familia. La mayor parte de las nuevas formas de interacción tiene lugar fuera del control directo de sus padres o del territorio. 
Durante la adolescencia el grupo de iguales que forman los adolescentes se convierte en una importante agencia de socialización, aprendiendo otro tipo de prácticas comportamentales regidas por sistemas contemporáneos formales e informales. Por ejemplo, otro espacio de la gran escuela que es la vida en comunidad y en organización; pero también es su espejo porque en él se refleja su verdadera cara, los sistemas contemporáneos de dominación que continúan ampliándose en la medida en que se van asentando en la vida adulta.

La socialización secundaria es, pues, un proceso por el cual se internalizan 'submundos' institucionales que contrastan con el "mundo de base" adquirido en la socialización primaria. Ahora entran en juego nuevos agentes de socialización tales como las instituciones ya sean laborales, políticas o religiosas. Aquí, el adolescente decide en qué sector social quiere introducirse e internalizar las reglas de juego que en este funcionan.

Tabla 1. Esquema comparativo de los tipos de socialización

\begin{tabular}{|c|c|c|}
\hline Socialización & Primaria & Secundaria \\
\hline Objetivos & $\begin{array}{l}\text { Convertir a un sujeto en } \\
\text { miembro de una sociedad } \\
\text { y desarrollar su identidad }\end{array}$ & $\begin{array}{l}\text { Adaptación del individuo ya } \\
\text { socializado a nuevos sectores } \\
\text { de su sociedad y desarrollar su } \\
\text { identidad }\end{array}$ \\
\hline Momentos & Niñez & $\begin{array}{l}\text { Posterior a la niñez en distintos } \\
\text { momentos a lo largo de la vida }\end{array}$ \\
\hline Espacio & Seno familiar & $\begin{array}{l}\text { Otras instituciones (escuela, } \\
\text { lugar de trabajo) }\end{array}$ \\
\hline Agentes & $\begin{array}{l}\text { Padres o tutores y demás } \\
\text { familiares (no posibilidad } \\
\text { de elección) }\end{array}$ & $\begin{array}{l}\text { Escuela, medios de } \\
\text { comunicación, instituciones } \\
\text { laborales, legales, políticas, } \\
\text { religiosas (posibilidad de } \\
\text { elección) }\end{array}$ \\
\hline $\begin{array}{l}\text { Tipo de } \\
\text { aprendizaje }\end{array}$ & $\begin{array}{l}\text { Aprendizaje con enorme } \\
\text { carga emocional/afectiva }\end{array}$ & $\begin{array}{l}\text { Aprendizaje con poca o ninguna } \\
\text { carga emocional/afectiva }\end{array}$ \\
\hline Proceso & Se parte de 'cero' & $\begin{array}{l}\text { Se parte de lo adquirido en la } \\
\text { socialización primaria }\end{array}$ \\
\hline Implantación & Muy firme & Menos firme que la primaria \\
\hline Resultado & $\begin{array}{l}\text { Se construye el "primer } \\
\text { mundo" del individuo }\end{array}$ & $\begin{array}{l}\text { Se adquiere conocimiento de } \\
\text { otros 'submundos' }\end{array}$ \\
\hline
\end{tabular}

Fuente: Contrera (2014). 
En términos generales definimos la socialización como el proceso por medio del cual el niño o la niña, formado en el seno de la tierra, aprende, recorre e interioriza, en el transcurso de su vida, los elementos socioculturales de su medio ambiente y los integra a la estructura de su ser bajo la influencia de experiencias y de agentes sociales significativos, adaptándose así al entorno social en cuyo seno debe vivir (Rocher, 1980). En este camino recorrido, el ser nasa retorna a su territorio tal y como lo proyecta la simbología del espiral $^{3}$.

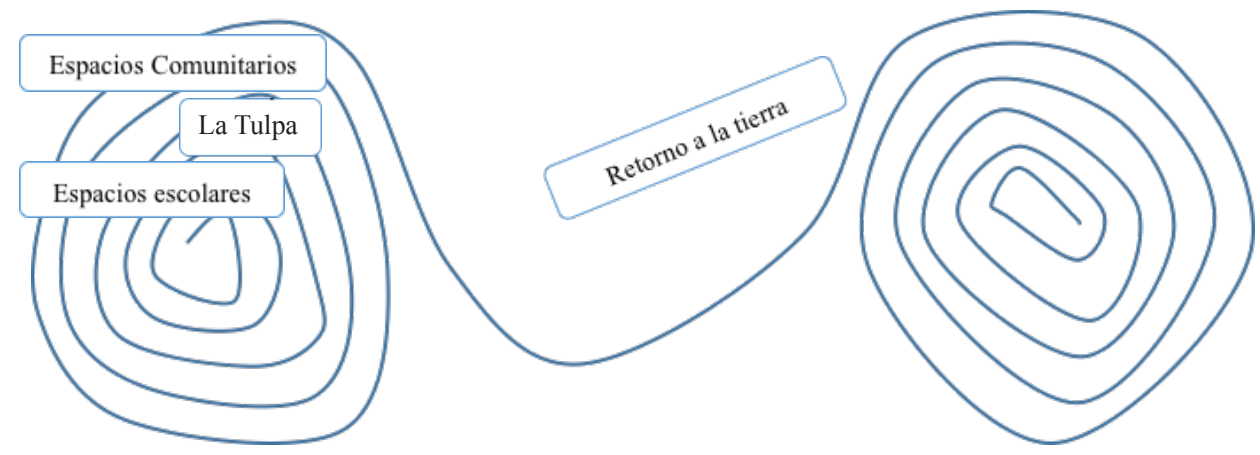

Figura 1. Cosmovisión nasa.

Fuente: elaboración propia de la autora.

A partir de la vivencia de los indígenas nasa, la educación ha sido base fundamental para la revitalización de las culturas y el mantenimiento de la identidad. Esta circunstancia ha significado enfrentar muchas situaciones que afectan la integridad y el pensamiento de cada pueblo generado por las condiciones de discriminación, desconocimiento y llegada de nuevas culturas que no se pueden desconocer. Desde las realidades en las que se desenvuelven hoy las semillas de vida ${ }^{4}$, entendidas como la generación de jóvenes, niños y niñas presentes en las comunidades, se expresa una tensión entre la tradición que se defiende y las nuevas prácticas culturales que acechan por doquier.

\footnotetext{
3 "La vida se conecta con el universo, el espiral nace de un punto y termina en otro, el paso que se da, como se viene planificando la vida". "Cuando hablamos de los cosmos o espiral aparece en la yema de los dedos, en los pies, los animales presentan el espiral o lo arboles. Todo se conecta con el espacio o habitantes de la naturaleza, los que nos hacen permanecer y retornar en el tiempo". Plan de Vida del Resguardo indígena de Mosoco.

${ }^{4}$ Los niños ocupan un lugar importante en la comunidad, teniendo en cuenta el género y los estadios o etapas de crecimiento que cada pueblo establece de manera particular. La relación con los miembros de la comunidad es determinada por los procesos de socialización especialmente en lo que se refiere a las formas de transmisión de los saberes y conocimientos los cuales determinan el rol que cada persona desempeña desde muy temprana edad; espacio que los va orientando hacia una manera especial de ver el mundo, comportarse, actuar y pensar (Perfil SEIP).
} 
Al ser este el punto de partida se pone sobre la mesa los procesos de socialización que emergen en las familias nasa, formadas tanto en la relación de padre y madre como la familia grande integrada por los abuelos, tíos, personas u otros seres que están cercanos biológica, cultural y espiritualmente ${ }^{5}$. Estos referentes familiares desarrollan en los niños lo afectivo, lo social, el aprendizaje del idioma nasa yuwe, las costumbres y otros elementos fundamentales para la construcción de la identidad cultural. Una noción que designa el movimiento mediante el cual la sociedad modela a los individuos que viven en su seno.

Ahora bien, no podemos desligarnos de la comunidad o pueblo en el que los niños y niñas se desenvuelven durante su primer ciclo de vida. Espacio de intercambio donde se "desarrollan valores, costumbres, comportamientos, saberes y muchos otros elementos de la convivencia cotidiana, que son fuente de la formación integral de las personas" ${ }^{16}$ (Guegia, 2010.p 13). En palabras de Luckmann (1927): “aprender la cultura y formar la identidad es como las dos caras de una misma moneda, y ambos constituyen el contenido básico del proceso de socialización" (p. ).

Tal vez existe una relación estrecha entre el contenido diario que los jóvenes realizan en la escuela, el colegio y otros espacios en los que converge su vida. Aquí es donde nacen las nuevas prácticas de identidad, que envuelta en el sentido colectivo se revierte en las familias que conforman los resguardos; las cuales reconocen y valoran los diferentes pueblos y culturas que conviven en el país y en la sociedad. En general se propende por una relación de equidad social, aceptación, respeto a la diferencia y armonía en la convivencia. Aunque hay un ciclo de tensión que pone en manos de las nuevas generaciones la necesidad de sobrevivir en un universo inmerso de mundos globalizantes y exigentes de adaptación.

\section{La política pública de infancia en Páez}

Siguiendo en la misma línea, se presenta ahora el recorrido y experiencia significativa desde la política pública de infancia y adolescencia del municipio de Páez (PPIA) ${ }^{7}$. Esta toma en cuenta el reconocimiento de elementos y dinámicas en marcha frente a los deseos e imaginarios con respecto a las estrategias que se deben implementar para mejorar la calidad de vida de las futuras generaciones; se incluyen elementos necesarios de

\footnotetext{
${ }^{5}$ Concepto de familia (Perfil SEIP).

${ }^{6}$ Concepto de comunidad (Perfil SEIP).

${ }^{7}$ La formulación de la PPIA “Los sueños y derechos de los niños nuestra mejor inversión" 2016-2027, se realizó con la participación de diferentes instituciones, buscando la construcción de un sueño y su concertación en torno a las situación de los niños y niñas de nuestro municipio.
} 
familias paeces (la educación, salud, ambientes sanos, convivencia, familias, vivienda digna, recreación, deporte y participación efectiva en distintos escenarios). Además, quiere aportar una lectura de las dinámicas cotidianas que se vienen teniendo en cuenta para la conformación de espacios vitales; pero también de los elementos que generan tensión con la dimensión étnicacultural de los actores.

\section{Experiencia desde la PPIA “Los sueños y derechos de los niños nuestra mejor inversión"}

La PPIA fue realizada en 9 zonas del municipio con actores sociales, autoridades y representantes de las instituciones. El ejercicio llevado a cabo, por la administración municipal de la época, fue aprobado en el 2015 para un período de 12 años. Esta expresa las situaciones deseadas por las semillas de vida evidenciando la experiencia dada desde las comunidades indígenas, afrodescendientes, campesinas y mestizas con las que se comienza con un pacto inicial, de donde se da a conocer sueños a futuro, partiendo de las realidades en las que se encuentran los habitantes, reconociendo la diversidad étnica que prevalece en el territorio; lo cual permite un enfoque diferencial en la formulación de la política, reconociendo la importancia de los tejidos culturales y estrategias políticas que se vienen poniendo en práctica para mejorar la calidad de vida de todo un municipio especialmente de las futuras generaciones.

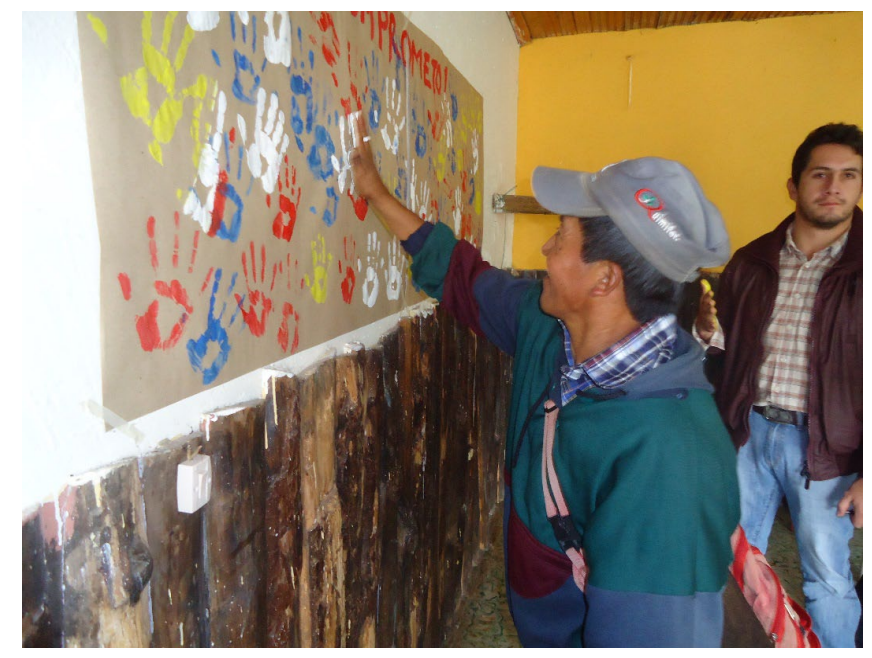

Figura 2. Autoridades de los resguardos indígenas, haciendo parte del pacto inicial de la formulación de la PPIA.

Fuente: trabajo de campo. 
A continuación, como fuente de información, se expresan los resultados a nivel general dados a través de la voz de los niños. Estos incluyen temáticas referentes a derechos fundamentales para los infantes, la vida, integridad física, salud seguridad social, alimentación equilibrada, nombre, nacionalidad, tener una familia y no ser separado de ella, el cuidado, el amor, la educación, la cultura, la recreación y la libre expresión de su opinión. Partiendo de una postura de requerimiento hacia el Estado dentro de las necesidades básicas contempladas en el artículo 44 de la Constitución.

De esta manera es necesario resaltar las estrategias puestas en marcha para el fortalecimiento de las prácticas de identidad nasa que familias e instituciones han venido impartiendo en los últimos años, siendo un tema en las agendas políticas de comuneros y líderes preocupados por salvaguardar los pensamientos y sabiduría ancestral enfrentados a sistemas modernistas.

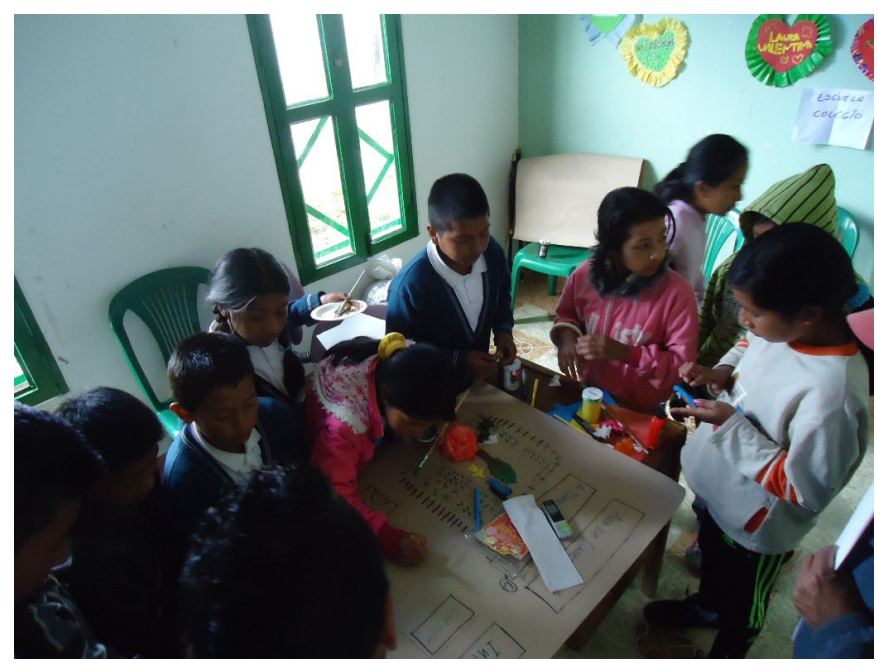

Figura 3. Dibujo realizado por los niños y niñas del resguardo de Mosoco. Fuente: trabajo de campo.

En este sentido el resguardo de Mosoco, comunidad participante de este proceso de construcción, también se une a los esfuerzos realizados por los comuneros $^{8}$; al dar cuenta de las restricciones y potencialidades que influyen en la identidad étnica nasa y de las subjetividades emergentes dadas desde el contexto familiar y/o comunitario rodeado de nuevos

\footnotetext{
${ }^{8}$ Persona que hace parte de una comunidad, para nuestro caso también de un censo o registro de un resguardo indígena.
} 
mundos de socialización; pero también de nuevas esperanzas en búsqueda de un buen vivir ${ }^{9}$ para todos.

Es así, que a través de la voz de las semillas de vida se le da relevancia a: la familia (vista desde el papá, mamá, hermanos y abuelos) como eje central de la formación inicial. La 'tulpa', lugar de encuentro y concentración de los principales aprendizajes; aprendizajes trasferidos mediante la trasmisión oral que los taitas y malulas hacen a sus hijos. Manejo y respeto por las plantas medicinales y cultivos propios de la región. La huerta o tul como un espacio donde comparten con su familia, pero a la vez aprenden a evitar daños ambientales. La autonomía alimentaria con consciencia ambiental; salvaguardando la madre tierra como medio que provee los recursos necesarios y conserva la vida de las especies, plantas medicinales, cultivos propios y sitios sagrados que posee el resguardo - mundo en el que se mueven los niños, conectándolos a un mundo espiritual que se imparte por los mayores y los médicos tradicionales (Kiwe The)-. Los Kiwe The permanecen en el territorio desde el mismo momento del nacimiento brindando los cuidados necesarios a través del manejo y uso de plantas medicinales; pero también desde el equilibrio natural que durante siglos ha permitido que sobrevivamos como pueblo nasa.

Así pues, traemos a la mesa a las comunidades indígenas de Colombia. Vislumbrando los medios, la naturaleza, la madre tierra y los mayores que son elementos fundamentales dentro del marco de la espiritualidad y la esencia de la lectura de las cosas. Esta lógica, inscrita en "un saber del cuerpo que no es pensable desde la conciencia en que se representa el mundo, pero que es accesible a la experiencia originaria en que se constituye el mundo" (Cabildo Indígena de Mosoco, 2015, p 25 ). Es decir que se simboliza a través de repertorios humanos que implican el sujeto-naturaleza.

En un lugar no muy lejano existe un bello paraíso con praderas, jardines, bosques, nacimientos de agua, sitios sagrados que dan color sentido a la defensa del territorio. Sus caminos conducen a los tules, parques, escuelas, colegios, hogares donde se refleja identidad cultural, amor, confianza, respeto. Sus gentes están organizadas en familias que cada semana salen con sus hijos a recrearse con tranquilidad y libertad. En las noches alrededor de la tulpa se congrega papá, mamá, abuelos e hijos al diálogo y enseñanza de las buenas costumbres.

Los adolescentes son quienes ayudan a cuidar los espacios verdes, en sus tiempos libres salen a las calles a formar las mentes con diferentes libros y comparten con sus amigos. Entre los jardines se ven niños y

\footnotetext{
${ }_{9}$ También llamado el Sumak Kawsay, quien rechaza una perspectiva unilineal de la historia (de Sousa Santos, 2006) recurriendo a la idea del nosotros (para el pensamiento ancestral el mundo es eminentemente colectivo).
} 
niñas que juegan, corren, cantan y ríen por sentirse sanos y fuertes. Esta región se caracteriza por ser unida, alegre, trabajadora, solidaria, cuando llegan personas de otras partes comparten lo que tienen y muestran sus riquezas y usos y costumbres.

Al salir de este bello lugar mágico uno se siente renovado y animado a inculcar esta bella experiencia donde se refleja la identidad, armonía y la calidad de vida en sus habitantes. (Cuento realizado durante la formulación de la PPIA)

Por tanto, los adultos (o los padres, abuelos y autoridad tradicional) plantean la importancia de potenciar la identidad étnica nasa a través de una enseñanza que promueva espacios de formación; que impliquen el fortalecimiento cultural, pensamiento ancestral y conservación de prácticas cotidianas en las que se incluye el acompañamiento de las familias e instituciones educativas y comunitarias dentro del fomento de la medicina tradicional, uso del nasa yuwe, consumo de alimentos sanos y limpios, elaboración de artesanías, fortalecimiento del cabildo escolar y participación de los niños en espacios comunitarios de labranza, mingas y otros.

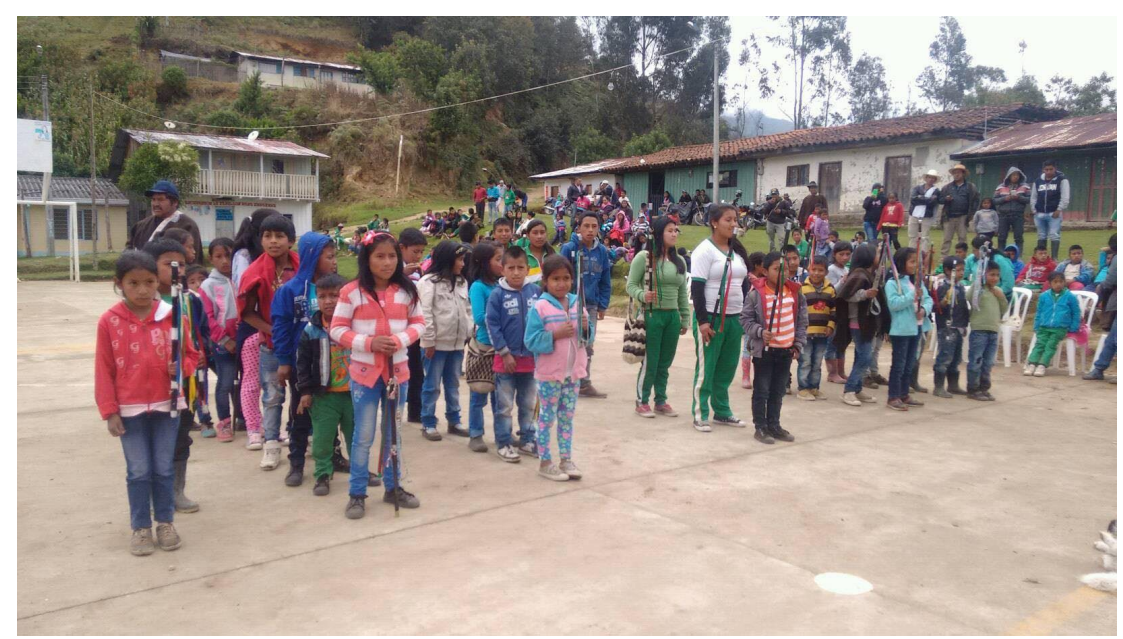

Figura 4. Posesión del cabildo escolar de la institución educativa Juan Tama, 2015. Fuente: trabajo de campo. 
Con lo dicho hasta el momento, los tejidos envueltos en una educación de fortalecimiento cultural se han venido entramando a través de escenarios marcados por los planes de vida que se construyen en el resguardo. Sin embargo expondremos algunas prácticas que ponen en tensión este argumento, dando cuenta de una realidad subjetiva que en los últimos años se construye en las semillas de vida.

\section{La subjetivación en la lectura de realidades}

Aunque existe un gran número de conductas rutinarias, los individuos también son capaces de producir acciones singulares que ponen a prueba su autonomía y su libertad en el seno de una división de trabajo compleja. Puesto que los valores son universales y 'racionales' ellos engendran una "intro-determinación", un fuero interno gracias al cual el personaje social no se corresponde totalmente con la subjetividad de cada uno (Riesman, 1964).

Acogiendo esta postura dialogaremos sobre una realidad inajenable, pero parte del crecimiento personal de jóvenes y niños quienes revelan en sus vivencias procesos de socialización múltiple; en donde se entreteje la influencia conjunta y a la vez contradictoria de la familia, atravesada por sociedades que conforman un individuo en función de las diferentes configuraciones sociales en las que está inserto.

De esta manera existen otros elementos que ponen en tensión un relieve esperanzador para las comunidades nasa, pues dan cuenta del desarrollo continuo que tiene el mundo moderno. No obstante, a partir del reconocimiento de las restricciones causantes de nuevas identidades que promueven intereses diversos en la población juvenil y en toda la comunidad en general. Las nuevas generaciones dialogan sobre estilos de vida basados en necesidades estandarizadas, saneamiento, vías pavimentadas, centros de capacitación, parques infantiles y un mundo digital que transita en su diario vivir. Igualmente buscan espacios de aceptación, adaptación de nuevas experiencias e inclusión de modas foráneas mezcladas con las problemáticas sociales del contexto. Ponen así en manos de padres de familia y autoridades, la reflexión de una educación que busca el acompañamiento cercano de sus hijos.

Un ejemplo de ello pueden ser las opiniones recogidas por los comuneros del resguardo de Mosoco sobre los conocimientos y habilidades a tener en cuenta para la garantía de los derechos de los niños, niñas y adolescentes: comunicación desde el vientre de la madre; rescatar el diálogo dentro de la familia; dedicarles mayor tiempo a los niños; que las madres lo sean cuando estén preparadas; no soltarles las riendas, ni darles 
mucha libertad a los hijos; educar con disciplina, comprometidos con los niños; descubrir y apoyar el proyecto de vida de los hijos; participar responsablemente de la escogencia de los profesores; mayor control, padres preparados y capacitados; enfrentar los problemas en conjunto para buscarle la solución a los problemas; apoyo profesional a la pareja para evitar la infidelidad, romper paradigmas.

En todos los casos la representación y participación de jóvenes en la propuesta educativa para el agenciamiento social, llevada a cabo en la institución educativa Juan Tama de Mosoco, describe algunas actuaciones tenidas en cuenta por los mismos actores; los cuales efectúan un proceso de diálogo en marcha, mingas de pensamiento, reflexionando desde las esferas de ser y hacer que convergen en las nuevas prácticas de identidad.

\section{Mingas de pensamiento en torno a las nuevas prácticas de identidad}

Las mingas de pensamiento, vistas como un elemento fundamental para la enseñanza y los aprendizajes desde la familia, se concentran ahora en los escenarios escolares envueltos en diálogos de saberes que incorporan experiencias e historias de vida en su formación académica y personal.

Sin duda, el elemento formal e informal de las mingas permitió el reconocimiento de nuevas prácticas de identidad; las cuales serán expuestas a continuación y tenidas en cuenta desde múltiples escenarios y marcados en el relacionamiento con otras culturas, con la sociedad mayoritaria y desde los ámbitos cotidianos.

En cuanto a los modelos de identidad festiva se destaca la inclusión de la Iglesia, subrayando las ritualidades religiosas: fiestas sampedrinas, decembrinas, cumpleaños (15 años), matrimonios entre otras. Esta educación en manos de la Iglesia ha sido desarrollada en el marco de las misiones - con una concepción pedagógica católica- convirtiéndose en el centro económico y de relaciones diversas, de aprovisionamiento y circulación de mercancías, de compra de fuerza de trabajo y comunicaciones.

Empero, la riqueza cultural encontrada en los diversos territorios difiere de esa idea de que los pueblos indígenas no tenían sus propios conocimientos. Por ello hasta la fecha se conservan ritualidades de festejo comunitario y personal: refrescamiento de varas, sembuch, posesión del cabildo mayor, rituales que conservan el legado espiritual de los mayores. 


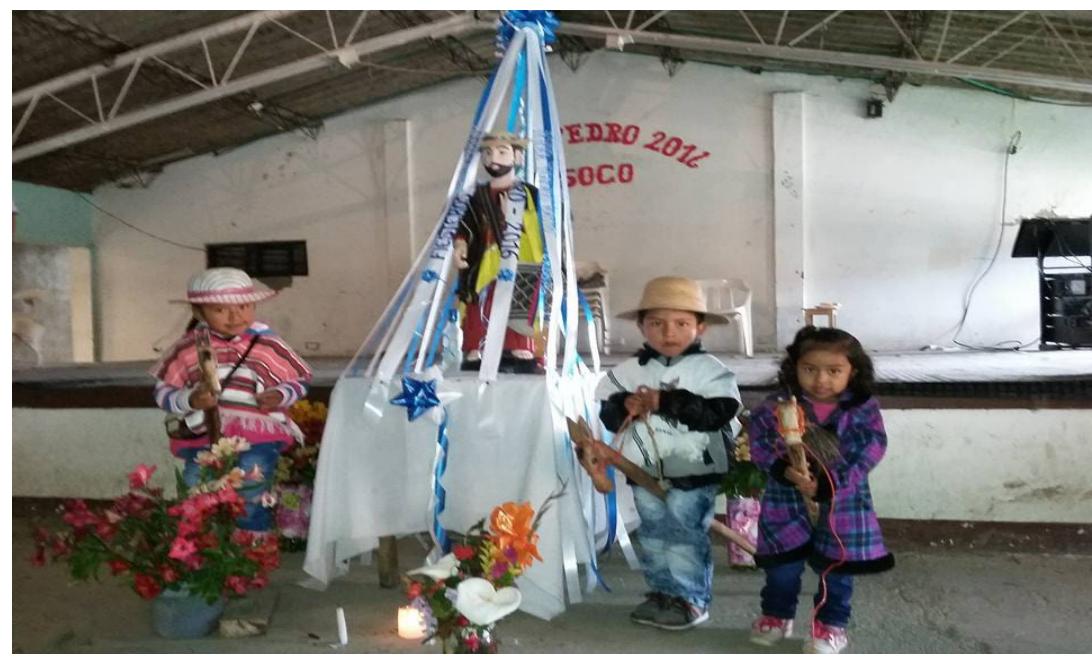

Figura 5. San Pedro y San Juan en el resguardo indígena de Mosoco, 2015. Fuente: trabajo de campo.

El reconocimiento directo en comunidad de las situaciones de la vida cotidiana, en tanto conductas como espacios y escenarios que son la expresión de concepciones estéticas propias de modelos occidentales. Esto refleja la inclusión de modas foráneas que jóvenes replican y absorben del encuentro dado en las grandes ciudades, acogidas en su paso por la universidad o centros de capacitación, creando nuevos estilos estéticos de mostrarse al mundo, apareciendo con gran destello el consumismo y la necesidad de medir su capacidad de existir en el mundo a través de las marcas de ropa que usan, las relaciones con el otro y por supuesto el abandono de la lengua materna y la espiritualidad que nos reconocen como nasa.

Lastimosamente, factores determinantes que han quedado relegados a un segundo plano: la espiritualidad en relación con la naturaleza y los rituales entre otros; sin reconocer la importancia dentro del desarrollo de los aprendizajes y enseñanzas propias para la interacción y crecimiento cultural, para establecer referentes más claros y profundos de identidad y en esa dimensión tener mejores posibilidades de establecer diálogos interculturales de reciprocidad y enriquecimiento mutuo.

En último lugar se muestra que el nuevo siglo, una colectividad del conocimiento a través de las TIC, se ha apoderado de todos los espacios de la vida cotidiana —en especial, de los jóvenes—; trayendo consigo un camino de competitividad y transformación desde esferas de relacionamiento y sistemas de educación. Sobre este particular hay dos posturas. 
Primero se reconoce un efecto benéfico de la tecnología al servicio de la educación, favoreciendo la adquisición de aprendizajes significativos desde el aprovechamiento de experiencias tecnológicas en las que se accede con mayor facilidad a los avances y conocimientos latentes fomentando la investigación, el reconocimiento de nuevos mundos y la exploración de escenarios por medio de una crítica reflexiva y la transformación de realidades.

Segundo la incorporación de redes digitales como Facebook, Whatsapp, Instagram, Twitter que han permeado la vida social de los jóvenes; convirtiéndose en la puerta de entrada, de incorporación de nuevas relaciones y con ello de procesos de identificación que revelan la aceptación del otro mediante la exposición de gustos y la necesidad de reconocimiento e intimidad al descubierto.

Estas perspectivas están ligadas a características de adaptación de las que tanto han luchado los pueblos indígenas hoy, pero de las que no se pueden limitar; sacándole provecho a nuevos medios tecnológicos que reconocen la orientación participativa y colectiva a través de las cuales se puede dar fuerza a los conocimientos que resisten, pero que deben encontrar en las nuevas prácticas de identidad una nueva forma de concebir el mundo que los rodea.

\section{Método}

El articulo centra su interés en identificar las restricciones y potencialidades generadas por los procesos de socialización de las nuevas generaciones en relación con la identidad étnica nasa y las subjetividades emergentes entre los y las jóvenes del resguardo indígena de Mosoco (Páez, Cauca), que permiten comprender nuevas miradas en torno a los planes de vida comunitario que giran en pro de toda una colectividad.

Debido a la naturaleza del fenómeno del proyecto puede mostrarse como un hecho reflexivo nutrido por los relatos autobiográficos de la autora, los sistemas de representación simbólica, actividades de la vida cotidiana y relación con el otro que proporcionan elementos de análisis dentro de la construcción identitaria del sujeto. En palabras de Contreras, "no vemos las cosas como son, sino como somos". Todas emergencias sociales que han provocado el surgimiento, la construcción, revitalización de nuevos actores y nuevas identidades sociales en niños y niñas que tienen en sus manos la garantía de la identidad cultural nasa del territorio. Que se enfrentan a estrategias de fortalecimiento comunitario, pero que no pueden desconocer las dinámicas actuales que se tejen alrededor del crecimiento personal de las juventudes impregnadas de nuevos procesos de subjetivación. 
En torno a lo anterior, el presente artículo es de corte cualitativo con un enfoque comprensivo. Existiendo un uso de la autobiografía como una herramienta complementaria debido a que permite el uso de los relatos significativos que realiza la autora como un elemento inicial de reflexión, pero que a la vez hace parte de las vivencias cotidianas del territorio; mostrando con ello elementos de construcción frente a los procesos de socialización que tejen episodios de construcción subjetiva, conocimiento del contexto y reflexiones sobre las dinámicas en marcha de la comunidad.

Por otro lado, es posible resaltar la relación con jóvenes y niños del resguardo permitiendo un mayor análisis de los sistemas de representación simbólica a través de las vivencias cotidianas de los actores protagonistas de esta lectura comprensiva con una mirada particular pero colectiva.

En relación con ello la búsqueda investigativa parte de la necesidad sentida por la autora que vislumbra de cerca la cotidianidad de la comunidad; inscrita en dinámicas de fortalecimiento cultural a través del plan de vida que, como lo decíamos anteriormente, requiere de la participación significativa de la voz de las nuevas generaciones.

Partiendo de un proceso simultáneo se genera una construcción con la propuesta educativa para el agenciamiento social (PEPAS), desarrollada en la institución educativa Juan Tama del resguardo indígena de Mosoco. En este sentido se da inicio a la propuesta con la socialización de la misma, con el fin de dar cuenta del proceso a seguir y recomendaciones dadas por el cuerpo docente de la institución. De esta manera se busca involucrar activamente a los padres y madres de familia en el proceso de formación de sus hijos, ya que al ser protagonistas del proceso de enseñanza-aprendizaje de estos se propone la realización de una serie de actividades: identificación, observación, exploración y discusión de una serie de temáticas que permiten comprender las prácticas de identidad en los procesos de socialización y subjetivación de las y los jóvenes. Finalmente se procesa la información recogida a partir del trabajo de campo nutrido de discursos, interacción, lenguajes y significados atribuidos a los mismos, para luego dar paso al análisis del mismo.

\section{Discusión}

El papel de las subjetividades y experiencias significativas en torno a la construcción identitaria del ser humano surge con gran fuerza. En este sentido se retoma en primera instancia la reflexión por medio del territorio, que pretende mostrar al lector la contextualización y algunos apartes influyentes dentro de las dinámicas comunitarias que se viven hoy 
en día; para esto se hace necesario el conocimiento y respeto por el otro a través de diálogos cercanos con jóvenes y padres de familia, otorgando una perspectiva de comprensión centrada en los propósitos comunitarios a partir de la voz de los mayores los cuales resaltan la trasmisión y adquisición de aprendizajes colectivos, reflexiones y vivencias del contexto principalmente en los primeros años de vida donde aún se tienen en cuenta las prácticas propias de la cultura nasa.

Sin embargo, existe una consciencia colectiva que resalta las vivencias y puntos de encuentro de nuevas generaciones que vislumbran formas de construcción

subjetiva mediante la correlación cercana con un mundo diverso y cargado de sistemas que modelan al sujeto de acuerdo a necesidades existentes en el medio; poniendo en tensión los lineamientos que hoy se trabajan en las comunidades indígenas en materia de educación y que están centrados en fortalecer lo propio, sin tener en cuenta las dinámicas y vivencias reflejadas por los jóvenes que requieren de espacios de participación donde sean tenidas en cuenta sus opiniones frente al tema de la identidad. Con esta idea, quizás el derecho a la participación del que tanto promulgan organismos internacionales y locales llegue a surtir efecto dentro de los procesos comunitarios; en este caso dentro de la construcción de los planes de vida donde los escenarios educativos, familiares y demás en los que se desenvuelven los niños son un argumento central del que se debe partir para comprender las nuevas prácticas de identidad de las nuevas generaciones.

Es bien sabido que si le damos el voto que se merecen los niños y jóvenes, será más eficaz el proceso que se desea implementar. Insistimos en que existe infinidad de subjetividades que rodean al niño y niña durante todo su crecimiento, por eso vemos necesario centrar los resultados esperados a partir del ir comunitario a través del pasado: el origen, las fuentes, a cómo era la situación antes según los mayores; pero también pensando en los pensamientos de hoy, esos que envuelven a las nuevas generaciones forjando una proyección hacia el futuro de las comunidades, basándonos en acuerdos mutuos y cargados de respeto hacia el pensamiento del otro.

Con el tema en el ambiente, finalizando el texto, se presentan los esfuerzos que se vienen tejiendo al interior del municipio que articula acciones con el ánimo de fortalecer la "participación de niños, niñas, adolescentes y sociedad civil" (política pública infancia niñez y adolescencia). Por ello se trabaja en la implementación de la "Mesa de primera infancia y adolescencia" como escenario de participación democrática y gestión compuesta por diferentes actores sociales que se integran y encuentran para fortalecer las interacciones, socializar aprendizajes, realimentar experiencias y potenciar la articulación sectorial, institucional y territorial. 


\section{Conclusiones}

Como se ha expresado a lo largo del artículo, resulta importante que las comunidades indígenas consideren la atención a las semillas de vida como agentes y actores sociales; no se trata solo de investigar sobre ellos, ni de diseñar políticas para su comunidad, se trata de convertirlos en los actores principales de reproducción cultural generando un sentido de participación a través del contexto familiar y comunitario. Para ello se necesita permitirles hablar y permitirnos escucharlos como parte de nuestra socialización cotidiana, aunque desde la diversidad de los espacios de socialización incluidos en los planes de vida que desarrolla el resguardo indígena de Mosoco.

No cabe duda de que es necesario abrir un lugar de encuentro que tenga como calzada replantear los lineamientos que vienen trazados por el sistema educativo indígena propio (SEIP), acogiendo las expectativas de un mundo pluricultural, partiendo desde un contexto diverso y afrontando las complejidades del mundo globalizante del presente que envuelve la subjetividad del individuo; así, es claro reconocer que las disposiciones de realidades sociales también tendrán acogida como una estrategia colectiva dentro de las necesidades de las nuevas generaciones.

\section{Referencias bibliográficas}

Arias, H. (2011). Territorio indígena kankuamo: proceso de reconfiguración del Resguardo desde las dimensiones socioculturales (Tesis de pregrado) Universidad Nacional de Colombia, Medellín, Colombia.

Alcaldía municipal Páez-Cauca (2016-2019). Política publica para la infancia, la niñez y la adolescencia del municipio de Páez, Belalcázar, Colombia.

Berger, Peter L. y Luckmann, Thomas (1995). La construcción social de la realidad. Buenos Aires. Amorrortu editores. Obra original publicada en 1967.

Boaventura, S. (2010) Descolonizar el Saber, reinventar el poder. Universidad la república, Montevideo, Uruguay.

Cabildo Indígena de Mosoco. (2006). Plan de vida en defensa, permanencia y autonomía del territorio nasa mosoqueño. Mosoco, Colombia: Institución educativa Juan Tama.

Cabildo Indígena de Mosoco. (2015). Plan de vida en defensa, permanencia y autonomía del territorio nasa mosoqueño. Mosoco, Colombia: Institución educativa Juan Tama.

Contreras, M. (2001) socialización y escuela Centro Universitario de Estudios Superiores Sagrada Familia - Úbeda

Guegia, C. (2010) Proceso Educativo de la escuela de Escalereta. Universidad del Cauca, Popayán, Colombia.

Guegia-Hurtado, J.P. (2017). Al paso de las semillas de vida del pueblo nasa de Tierradentro y la construcción de las nuevas identidades. Revista de Antropología y Sociología: VIRAJES, 19 (2), 07 de junio-2017. 
Hernández, E. (2006). La resistencia civil de los indígenas del cauca. Scientific Electronic Library Online ,11 (1), 66-79.

Juárez, A. (2006). Autobiografía, identidad y ropa en el siglo de oro. A, Rowe (Ed), El cuerpo vestido y la construcción de la identidad en las narrativas autobiográficas del siglo de oro (pp.1230). Tamesis, Woodbridge.

Lockwood-David. (1964). El paradigma de sistemas: posibilidades para una práctica social emancipadora. Revista Filosofía Universitaria Costa Rica, XXVII (66), 387-398.

Luckmann-Thomas y Berger Peter (2003) La construcción social de la realidad. Amorrortu editores S. A., Paraguay 1225, 7" piso (1057) Buenos Aires.

Riesman, D. (1950) La muchedumbre solitaria un estudio sobre el carácter norteamericano. Buenos aires, Argentina.

Rocher, G. (1980). Introducción a la Sociología General. Barcelona, Herder. 7ª ed. 